\title{
Global Null Controllability of the One-Dimensional Nonlinear Slow Diffusion Equation
}

\author{
Jean-Michel Coron, ${ }^{*} \quad$ Jesús Ildefonso Díaz, ${ }^{\dagger}$ \\ Abdelmalek Drici, ${ }^{\ddagger} \quad$ Tommaso Mingazzini ${ }^{\S}$
}

Dedicated to Jacques-Louis Lions with the souvenir of his mastery and creativity

\begin{abstract}
We prove the global null controllability for the one-dimensional nonlinear slow diffusion equation by using both a boundary and an internal control. We assume that the internal control is only time dependent. The proof relies on the "return method" in a combination of some local controllability results for non-degenerate equations and rescaling techniques. Keywords: Nonlinear control, nonlinear slow equation, porous medium equation
\end{abstract}

\section{Introduction}

We study the null controllability of the one-dimensional nonlinear slow diffusion equation, sometimes referred as the Porous Media Equation (shortly PME), by using a combination of internal and boundary controls. Our techniques of proof need such a combination of both controls due to the degenerate nature of this quasilinear parabolic equation.

The PME belongs to the more general family of non linear diffusion equations of the form

$$
y_{t}-\Delta \phi(y)=f,
$$

*Institut universitaire de France and Université Pierre et Marie Curie (Paris 6), UMR 7598 Laboratoire Jacques-Louis Lions, 4, place Jussieu, F-75252 Paris cedex 5 France (coron@ann.jussieu.fr).

†Universidad Complutense de Madrid, Instituto de Matemática Interdisiplinar and Dpto. de Matemática Aplicada, Plaza de las Ciencias 3, 28040 Madrid, España -

(diaz.racefyn@insde.es).

$\ddagger$ Université Pierre et Marie Curie (Paris 6), UMR 7598 Laboratoire Jacques-Louis Lions, 4, place Jussieu, F-75252 Paris cedex 5 France -

(drici@ann.jussieu.fr).

$\S$ Universidad Complutense de Madrid, Dpto. de Matemática Aplicada, 28040 Madrid, España -

(tommaso.mingazzini@gmail.com). 
where $\phi$ is a continuous non-decreasing function with $\phi(0)=0$. For the PME, the constitutive law is precisely given by

$$
\phi(y)=|y|^{m-1} y,
$$

with $m \geq 1$.

This family of equations arises in many different frameworks and, depending on the nature of $\phi$, it models different diffusion processes, mainly grouped into three categories, namely "slow diffusion", "fast diffusion" and linear processes.

The "slow diffusion" case is characterized by a finite speed of propagation and the formation of free boundaries while the "fast diffusion" one is characterized by the finite extinction time, which means that the solution becomes identically zero after a finite time.

If one neglects the source term, i.e. $f=0$, and imposes the constraint of nonnegativeness to the solutions, something which is fundamental in all the applications where $y$ represents for example any density, then one can precisely characterize these phenomena. In fact, it was shown in [12] that the homogeneous Dirichlet problem associated to 1.1 on a bounded open set $\Omega$ of $\mathbb{R}^{N}$ satisfies the finite extinction time if and only if

$$
\int_{0}^{1} \frac{d s}{\phi(s)}<+\infty,
$$

which, for constitutive laws given by 1.2$)$, corresponds to the case $m \in(0,1)$. If, on the contrary

$$
\int_{0}^{1} \frac{d s}{\phi(s)}=+\infty,
$$

(which is the case of $m \geq 1$ ) then, for any initial datum $y_{0} \in H^{-1}(\Omega) \cap L^{1}(\Omega)$ with $(-\Delta)^{-1} y_{0} \in L^{\infty}(\Omega)$, it occurs a kind of "retention property". This means that, if $y_{0}(x)>0$ on a positively measured subset $\Omega^{\prime} \subset \Omega$, then $y(t,)>$.0 on $\Omega^{\prime}$ for any $t>0$. If, in addition to $[1.3$, we have that $\phi$ satisfies that

$$
\int_{0}^{1} \frac{\phi^{\prime}(s) d s}{s}<+\infty,
$$

(i.e. $m>1$ in the case of 1.2 ) then the solution enjoys the finite speed of propagation and gives rise to a free boundary given by the boundary of its support $(\partial\{y>0\})$.

Most typical applications of "slow diffusion" are the nonlinear heat propagation, groundwater filtration and the flow of an ideal gas in a homogeneous porous medium (from which the name PME). The fast diffusion, instead, finds a paradigmatic application to the flows in plasma physics. Many results and references can by found in the monographs [2] and [22].

As already said, the main aim of this paper is to show how a combined action of boundary controls and a spatially homogeneous internal control may allow the global extinction of the solution (the so called global null controllability) in any prescribed temporal horizon $T>0$. In this paper we shall prove the global null controllability for the following two control problems

$$
P_{D D}\left\{\begin{array}{lr}
y_{t}-\left(y^{m}\right)_{x x}=u(t) \chi_{I}(t) & \text { in }(0,1) \times(0, T), \\
y(0, t)=v_{0}(t) \chi_{I}(t) & t \in(0, T), \\
y(1, t)=v_{1}(t) \chi_{I}(t) & t \in(0, T), \\
y(x, 0)=y_{0}(x) & x \in(0,1),
\end{array}\right.
$$


and

$$
P_{D N}\left\{\begin{array}{lr}
y_{t}-\left(y^{m}\right)_{x x}=u(t) \chi_{I}(t) & \text { in }(0,1) \times(0, T), \\
\left(y^{m}\right)_{x}(0, t)=0 & t \in(0, T), \\
y(1, t)=v_{1}(t) \chi_{I}(t) & t \in(0, T), \\
y(x, 0)=y_{0}(x) & x \in(0,1),
\end{array}\right.
$$

where $I=\left(t_{1}, T\right)$ with $t_{1} \in(0, T), m \geq 1$ and $\chi_{I}$ is the characteristic function of $I$. In both problems, $y$ represents the state variable and $U_{D N}:=\left(u \chi_{I}, v_{1} \chi_{I}\right)$ and $U_{D D}=\left(u \chi_{I}, v_{0} \chi_{I}, v_{1} \chi_{I}\right)$, respectively, are the control variables. The function $y^{m}$ should be more properly written in form 1.2 , but as we shall impose the constraint $y \geq 0$ it makes no real difference.

We point out the fact that the internal control $u(t)$ have the property to be independent of the space variable $x$ and that the controls are active just on a part and not on all the time interval. Moreover, since, as we shall show, the systems are null controllable in any arbitrarily fixed time, the localized form of the control $u(t) \chi_{I}(t)$ (and the same for the boundary controls), on a subinterval of $[0, T]$, is more an emphatic than a real difficulty. It serves mostly to underline the fact that, in our choice, the control are not active in the first time lapse. In the same way it could be possible to take a control interval $(\underline{t}, \bar{t})$ with $\underline{t}, \bar{t} \in(0, T)$ or even more generally three different control intervals, one for each control $v_{0}, v_{1}, u$, such that the intersection of the three intervals is not empty.

The main results of this paper are contained in the following statement.

Theorem 1.1. Let $m \in[1,+\infty)$.

i) For any initial data $y_{0} \in H^{-1}(0,1)$ such that $y_{0} \geq 0$ and any time $T>0$, there exist controls $v_{0}(t), v_{1}(t)$ and $u(t)$ with $v_{0}(t) \chi_{I}(t), v_{1}(t) \chi_{I}(t) \in H^{1}(0, T)$, $v_{0}, v_{1} \geq 0$ and $u \in L^{\infty}(0, T)$ such that the solution $y$ of $P_{D D}$ satisfies $y \geq 0$ on $(0,1) \times(0, T)$, and $y(\cdot, T)=0$ on $(0,1)$.

ii) For any initial data $y_{0} \in H^{-1}(0,1)$ such that $y_{0} \geq 0$ and any time $T>0$, there exist controls $v_{1}(t)$ and $u(t)$ with $v_{1}(t) \chi_{I}(t) \in H^{1}(0, T), v_{1} \geq 0$ and $u \in$ $L^{\infty}(0, T)$ such that the solution $y$ of $P_{D N}$ satisfies $y \geq 0$ on $(0,1) \times(0, T)$, and $y(\cdot, T)=0$ on $(0,1)$.

Notice that since $H^{-1}(0,1)=\left(H_{0}^{1}(0,1)\right)^{\prime}$ and $H_{0}^{1}(0,1) \subset C([0,1])$ then we have that $H^{-1}(0,1) \supset \mathcal{M}(0,1)$, the set of bounded Borel measures on $(0,1)$. Hence, the initial datum can be, for instance, a Dirac mass distribution at a point in $(0,1)$. As said before, in the case of slow diffusion, $m>1$, which is the one we are dealing with, the solution may present a free boundary given by the boundary of its support (once that the support of $y_{0}$ is strictly smaller than $[0,1])$. Nevertheless, our method of proof is based in the strategy to avoid such a free boundary. Indeed, on the set of points $(x, t)$ where $y$ vanishes (i.e. on the points $(x, t) \in(0,1) \times(0, T) \backslash \operatorname{supp}(y))$ the diffusion operator is not differentiable at the function $y \equiv 0$ and so some linearization methods which works quite well for second order semilinear parabolic problems (see, e.g., [13, 17, 19, 20]) can not be directly applied. Moreover, the evanescent viscosity perturbation with some higher order terms only gives some controllability results for suitable functions $\phi$ as the ones of the Stefan problem ([13, [14] and [15]). 
Here we shall follow a different approach which is based mainly on the so called return method introduced in [9, 10, (see [11, Chapter 6] for more information on this method). More precisely, we shall prove first the null controllability of problem (1.4) by applying an idea appeared in [8] (for the controllability of the Burgers equation), and then, in a second step, by using some symmetry arguments we shall show that the same result holds in fact for 1.5 .

Our version of the return method consists in the idea of choosing a suitable parametrized family of trajectories $a(t) / \varepsilon$, which is independent of the space variable, going from the initial state $y \equiv 0$ to the final state $y \equiv 0$. Then we shall use the controls to reach one of such trajectories, no matter which one, in some concrete large positive time smaller then the final $T$. Once we prescribe a partition, of the form $0<t_{1}<t_{2}<t_{3}<T$, we shall choose a function $a(t)$ satisfying the following properties:

i) $a \in C^{2}([0, T])$;

ii) $a(t)=0,0 \leq t \leq t_{1}$ and $t=T$;

iii) $a(t)>0, t \in\left(t_{1}, T\right)$;

iv) $a(t)=1, t_{2} \leq t \leq t_{3}$.

Then, we can write the decomposition of the solution $y$ of problem $P_{D D}$ as a perturbation of the explicit solution $a(t) / \varepsilon$ of the same equation with the controls $U=(a(t) / \varepsilon, a(t) / \varepsilon, a(t) / \varepsilon)$ in the following way:

$$
y(x, t)=\left(\frac{a(t)}{\varepsilon}+z(x, t)\right) .
$$

Our aim is now to find controls such that $z\left(x, t_{3}\right) \equiv 0$, which means that we have controlled our solution $y(x, t)$ to the state $1 / \varepsilon$ at time $t=t_{3}$; this will be done by using a slight modification of a result in [4. On the final time interval $\left(t_{3}, T\right)$, we shall use the same trajectory, $y(x, t)=a(t) / \varepsilon$, to reach the final state $y(x, T) \equiv 0$. An ideal representation of the trajectory can be seen in Figure 1 .

One can see that the central core of our procedure is to drive the initial state to a constant state in a finite time thanks to the use of a boundary and internal control (which depends only on the time variable).

On the first interval $\left(0, t_{1}\right)$ we shall not make any use of the controls. So we let the solution, $y(t):=y(., t)$, to regularize itself from an initial state in $H^{-1}(0,1)$ to a smoother one in $H_{0}^{1}(0,1)$ for $t=t_{1}$. Then, as the degenerate character of the diffusion operator neglects the diffusion effects outside the support of the state, we move $y(t)$ away from the zero state by asking $z(t)=z(., t)$ to be non-negative at least in the interval $\left(t_{1}, t_{2}\right)$. With this trick, the solution $y(t)$ will be far enough from zero. On the interval $\left(t_{2}, t_{3}\right)$ the states $y(t)$ will be kept strictly positive even if the internal control $u(t)$ will be allowed to take negative values.

As already mentioned concerning the local retention property, we point out that the presence of the control $u(t)$ is fundamental for the global null controllability property. To be more precise, notice that if we assume $u(t) \equiv 0$ then we can find initial states which cannot be steered to zero at time $T$ just with some non negative boundary controls. As a matter of fact, to show it, one can 


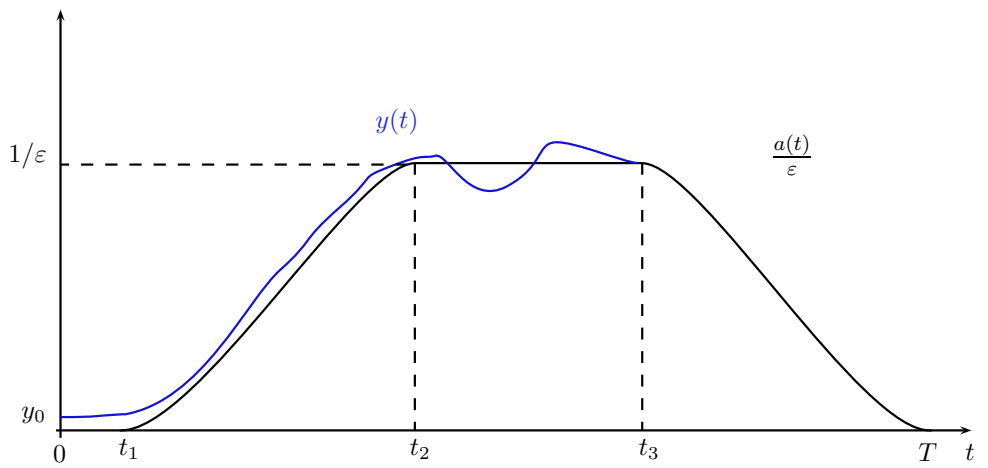

Figure 1: Solution profile.

use the well-known family of Barenblatt solutions [3, 22. (also known as ZKB solutions). Indeed, if we introduce the parameters

$$
\alpha=\frac{1}{m+1}, k=\frac{m-1}{2 m(m+1)}, \tau<<1
$$

and choose $C$ such that $(C / k)^{1 / 2}(T+\tau)^{\alpha}<1 / 2$, then the function

$$
y_{m}(x, t)=(t+\tau)^{-\alpha}\left(C-k|x-1 / 2|^{2}(t+\tau)^{-2 \alpha}\right)_{+}^{1 /(m-1)}
$$

is a solution of system 1.4 with $u=0, v_{0}=v_{1}=0$ and $y_{m}(x, T) \neq 0$. Any other solution of system (1.4) with the same initial data and $v_{0}, v_{1} \geq 0$ would be a supersolution of $y_{m}$ which implies that $y_{m}(x, 0)$ cannot be connected with $y(., T) \equiv 0$.

\section{Well posedness of the Cauchy problem}

For the existence theory of problem $(1.4)$ we refer to [1, 6, 7, 5, 21, 22]; in particular we adopt a frame similar to the ones in [1] and [6]. More precisely, we use the following definition.

Definition 2.1. Let $\left(v_{0}, v_{1}\right) \in L^{\infty}(0, T)^{2}$ and $v_{D}=(1-x) v_{0}(t)+x v_{1}(t)$ and let $u \in L^{\infty}(0, T)$. Assume that $y_{0} \in H^{-1}(0,1)$. We say that $y(x, t)$ is a weak solution of

$$
P_{D D} \begin{cases}y_{t}-\left(|y|^{m-1} y\right)_{x x}=u(t) & \text { in }(0,1) \times(0, T) \\ y(0, t)=v_{0}(t) & t \in(0, T) \\ y(1, t)=v_{1}(t) & t \in(0, T) \\ y(x, 0)=y_{0}(x) & x \in(0,1)\end{cases}
$$

if

$$
\begin{gathered}
y \in C^{0}\left([0, T] ; H^{-1}(0,1)\right) \text { and } y(0)=y_{0} \text { in } H^{-1}(0,1), \\
y \in L^{\infty}\left(\tau, T ; L^{1}(0,1)\right), \forall \tau \in(0, T], \\
\partial_{t} y \in L^{2}\left(\tau, T ; H^{-1}(0,1)\right), \forall \tau \in(0, T], \\
|y|^{m-1} y \in\left|v_{D}\right|^{m-1} v_{D}+L^{2}\left(\tau, T ; H_{0}^{1}(0,1)\right), \forall \tau \in(0, T],
\end{gathered}
$$


and if

$$
\begin{aligned}
\int_{\tau}^{T}<\partial_{t} y, \xi>+\int_{\tau}^{T} & \int_{0}^{1}\left(|y|^{m-1} y\right)_{x} \xi_{x}= \\
& \int_{\tau}^{T} \int_{0}^{1} u \xi, \forall \tau \in(0, T), \forall \xi \in L^{2}\left(0, T ; H_{0}^{1}(0,1)\right),
\end{aligned}
$$

where the symbol $\langle\cdot, \cdot\rangle$ stands for the dual pairing between $H^{-1}(0,1)$ and $H_{0}^{1}(0,1)$;

Remark 2.2. We have modified the definition of weak solution given in [1] in order to handle the case where $y_{0}$ is only in $H^{-1}(0,1)$, instead of $y_{0} \in L^{m+1}(0,1)$ as assumed in [1].

The modifications to extend the previous definition to the case of problem $\left(P_{N D}\right)$ are straightforward (see [1]). For instance, the extension to the interior of the boundary datum can be taken now as $v_{D}=\left(c_{1}+c_{2} x^{2}\right) v_{1}(t)$.

With this definition, one has the following proposition.

Proposition 2.3. The boundary-value problem 1.4 has at most one weak solution.

The proof of proposition 2.3 is the same as in [1, Theorem 2.4] due to the regularizing effect required in Definition 2.1. See also [5].

The next two propositions follow from results which can be found in [1, Theorem 1.7, Theorem 2.4] and [7.

Proposition 2.4. Suppose that $\left(v_{0}, v_{1}\right) \in H^{1}(0, T)^{2}$ and vanishes in a neighbourhood of $t=0$, then there exists one and only one weak solution of problem (1.4). Moreover, if

Proposition 2.5. Suppose that $\left(v_{0}, v_{1}\right) \in H^{1}(0, T)^{2}$ and that $y_{0} \in L^{m+1}$, then there exists one and only one weak solution y of problem 1.4. Moreover this solution satisfies

$$
\begin{gathered}
y \in L^{\infty}\left(0, T ; L^{1}(0,1)\right) \\
\partial_{t} y \in L^{2}\left(0, T ; H^{-1}(0,1)\right), \\
|y|^{m-1} y \in\left|v_{D}\right|^{m-1} v_{D}+L^{2}\left(0, T ; H_{0}^{1}(0,1)\right) .
\end{gathered}
$$

We now emphasize that the solution of problem $\left(P_{D D}\right)$ enjoys an additional semigroup property (we will need it in constructing the final trajectory), which directly follows from Definition 2.1. Proposition 2.4 and Proposition 2.5

Lemma 2.6 (Matching). Suppose that $y_{1}$ is a weak solution of (1.4) on the interval $\left(0, T_{1}\right)$ and that $y_{2}$ is a weak solution of 1.4 on the interval $\left(T_{1}, T\right)$ with $y_{2}\left(T_{1}\right)=y_{1}\left(T_{1}\right) \in L^{2}(0,1)$. If we denote

$$
y(t)= \begin{cases}y_{1}(t) \quad t \in\left(0, T_{1}\right), \\ y_{2}(t) \quad t \in\left(T_{1}, T\right),\end{cases}
$$

then $y$ is a weak solution of (1.4) on the interval $(0, T)$. 


\section{Proof of the main theorem: first step}

In the interval $\left(0, t_{1}\right]$ the solution with no controls evolves as in 7 , hence $0 \leq$ $y^{m}(t) \in H_{0}^{1}(0,1)$ for all $t \in\left(0, t_{1}\right]$. Due to the inclusion $H_{0}^{1}(0,1) \subset L^{\infty}(0,1)$ we got that $y_{1}(x):=y\left(x, t_{1}\right)$ is a bounded function. We call the solution on this first interval $y^{0}(x, t)$, i.e.

$$
y_{\mid\left(0, t_{1}\right)}=y^{0} .
$$

In order to be able to apply the null controllability result in [4] to the function $z(x, t)$, given in the decomposition $(1.6)$, on the interval $\left(t_{2}, t_{3}\right)$ we need the $H^{1}$ norm of $z\left(t_{2}\right)$ to be small enough. We want to find some estimates of the solution $z$ of

$$
\begin{cases}z_{t}-\left(m\left(\frac{a(t)}{\varepsilon}+z\right)^{m-1} z_{x}\right)_{x}=0, & \text { in }(0,1) \times\left(t_{1}, t_{2}\right), \\ z_{x}(t, 0)=z_{x}(t, 1)=0, & t \in\left(t_{1}, t_{2}\right), \\ z(x, 0)=y_{1}(x), & x \in(0,1) .\end{cases}
$$

For the existence, regularity and comparison results for this problem we refer to 18 , where the equation in recast in the form $\left(|Y|^{1 / m} \operatorname{sign}(Y)\right)_{t}-Y_{x x}=a^{\prime} / \varepsilon$. From the maximum principle, we deduce that $y_{1} \in L^{\infty}(0,1)$ and $y_{1} \geq 0$ imply that $z \in L^{\infty}\left((0,1) \times\left(t_{1}, t_{2}\right)\right)$ and $z \geq 0$. In fact, we have $0 \leq z \leq M$, where $M:=\left\|y_{1}\right\|_{L^{\infty}(0,1)}$ is a solution of the state equation of 3.2 , and in particular a super solution of 3.2 .

To study the behaviour of $z$, we will actually make use of rescaling.

\subsection{Small initial data and a priori estimates}

For $\delta>0$, we define $\tilde{z}:=\delta z$. Then $\tilde{z}$ satisfies

$$
\begin{cases}\tilde{z}_{t}-\left(m\left(\frac{a(t)}{\varepsilon}+\frac{1}{\delta} \tilde{z}\right)^{m-1} \tilde{z}_{x}\right)_{x}=0, & \text { in }(0,1) \times\left(t_{1}, t_{2}\right), \\ \tilde{z}_{x}(t, 0)=\tilde{z}_{x}(t, 1)=0, & t \in\left(t_{1}, t_{2}\right), \\ \tilde{z}(x, 0)=\delta y_{1}, & x \in(0,1) .\end{cases}
$$

After collecting the factor $\frac{1}{\varepsilon}$ and rescaling the time $\tau:=\frac{t}{\varepsilon^{m-1}}$, we get

$$
\tilde{z}_{t}-\left(m\left(a(\tau)+\frac{\varepsilon}{\delta} \tilde{z}\right)^{m-1} \tilde{z}_{x}\right)_{x}=0 .
$$

Choosing $\delta:=\varepsilon^{1-\alpha}$ with $0<\alpha<1$, the system can be written in the following form

$$
\begin{cases}\tilde{z}_{\tau}-\left(m\left(a(\tau)+\varepsilon^{\alpha} \tilde{z}\right)^{m-1} \tilde{z}_{x}\right)_{x}=0, & \text { in }(0,1) \times\left(\tau_{1}, \tau_{2}\right), \\ \tilde{z}_{x}(\tau, 0)=\tilde{z}_{x}(\tau, 1)=0, & \tau \in\left(\tau_{1}, \tau_{2}\right) \\ \tilde{z}(x, 0)=\varepsilon^{1-\alpha} y_{1}, & x \in(0,1)\end{cases}
$$

where $\tau:=\frac{t}{\varepsilon^{m-1}}$. For simplicity, we take $\alpha=1 / 2$. 
Thus, the null controllability of system 3.2 is reduced to the null controllability of system (3.4). As we can see, the initial data in (3.4) is now depending on $\varepsilon$ and tends to 0 as $\varepsilon \rightarrow 0$.

\section{$3.2 \quad H^{1}$-estimate}

We recall that, according to regularity theory for linear parabolic equations with bounded coefficients, $\tilde{z}(t) \in H^{2}(0,1)$ for $t>0$, see, e.g. [16, pp. 360-364] . Multiplying by $\tilde{z}_{x x}$ the first equation of (3.4) and integrating on $x \in(0,1)$, we get

$$
\int_{0}^{1} \tilde{z}_{\tau} \tilde{z}_{x x} d x=\int_{0}^{1}\left(m(a(\tau)+\sqrt{\varepsilon} \tilde{z})^{m-1} \tilde{z}_{x}\right)_{x} \tilde{z}_{x x} d x .
$$

Then, integrating by parts and using the boundary condition in (3.4), we are led to

$$
\begin{aligned}
\frac{1}{2 m} \frac{d}{d \tau} \int_{0}^{1} \tilde{z}_{x}^{2} d x=-\int_{0}^{1}(a(\tau)+\sqrt{\varepsilon} \tilde{z})^{m-1} \tilde{z}_{x x}^{2} d x \\
\quad-\frac{(m-1)}{3} \sqrt{\varepsilon} \int_{0}^{1}(a(\tau)+\sqrt{\varepsilon} \tilde{z})^{m-2}\left(\tilde{z}_{x}^{3}\right)_{x} d x \\
=-\int_{0}^{1}(a(\tau)+\sqrt{\varepsilon} \tilde{z})^{m-1} \tilde{z}_{x x}^{2} d x \\
+\frac{(m-1)(m-2)}{3} \varepsilon \int_{0}^{1}(a(\tau)+\sqrt{\varepsilon} \tilde{z})^{m-3} \tilde{z}_{x}^{4} d x .
\end{aligned}
$$

We denote by

$$
\begin{aligned}
& I T_{1}:=-\int_{0}^{1}(a(\tau)+\sqrt{\varepsilon} \tilde{z})^{m-1} \tilde{z}_{x x}^{2} d x \\
& I T_{2}:=\frac{(m-1)(m-2)}{3} \varepsilon \int_{0}^{1}(a(\tau)+\sqrt{\varepsilon} \tilde{z})^{m-3} \tilde{z}_{x}^{4} d x .
\end{aligned}
$$

We observe that $I T_{1} \leq 0$. Let us look to the term $I T_{2}$. For $m \in(1,2)$, we have that $I T_{2} \leq 0$. Otherwise,

$$
I T_{2} \leq \frac{(m-1)(m-2)}{3}\left(a(\tau)+\sqrt{\varepsilon}\|\tilde{z}\|_{\infty}\right)^{m-3} \varepsilon \int_{0}^{1} \tilde{z}_{x}^{4} d x .
$$

The fact that $L^{\infty}$ norm of $\tilde{z}$ is finite comes from the fact that $\tilde{z}=\delta z$ and that the supremum of $z$ is bounded, as already pointed out. We use now a well-known Gagliardo-Nirenberg's inequality in the case of a bounded interval:

Lemma 3.1. Suppose $z \in L^{\infty}(0,1)$ with $z_{x x} \in L^{2}(0,1)$ and either $z(0)=$ $z(1)=0$ or $z_{x}(0)=z_{x}(1)=0$, then

$$
\left\|z_{x}\right\|_{L^{4}} \leq \sqrt{3}\left\|z_{x x}\right\|_{L^{2}}^{\frac{1}{2}}\|z\|_{L^{\infty}}^{\frac{1}{2}} .
$$

Proof of lemma 3.1. Integrating by parts and using the boundary conditions, we obtain

$$
\int_{0}^{1} z_{x}^{4} d x=\int_{0}^{1} z_{x}^{3} z_{x} d x=-3 \int_{0}^{1} z_{x}^{2} z_{x x} z d x
$$


Then, using Cauchy-Schwarz's inequality, we get

$$
\left\|z_{x}\right\|_{L^{4}}^{4} \leq 3\left\|z_{x}\right\|_{L^{4}}^{2}\|z\|_{L^{\infty}}\left\|z_{x x}\right\|_{L^{2}}
$$

and the result follows immediately.

Setting $C^{\prime}:=C\|\tilde{z}\|_{L^{\infty}}^{2}$ and considering that $\left\|\tilde{z}_{x}\right\|_{L^{4}}^{4} \leq C^{\prime}\left\|\tilde{z}_{x x}\right\|_{L^{2}}^{2}$, we have

$$
\begin{aligned}
& \frac{1}{2 m} \frac{d}{d \tau} \int_{0}^{1} \tilde{z}_{x}^{2} d x \leq-\int_{0}^{1}(a(\tau)+\sqrt{\varepsilon} \tilde{z})^{m-1} \tilde{z}_{x x}^{2} d x+\frac{(m-1)(m-2)}{3}\left(a(\tau)+\sqrt{\varepsilon}\|\tilde{z}\|_{\infty}\right)^{m-3} \varepsilon \int_{0}^{1} \tilde{z}_{x}^{4} d x, \\
& \leq-(a(\tau))^{m-1} \int_{0}^{1} \tilde{z}_{x x}^{2} d x \\
& \quad+C^{\prime} \frac{(m-1)(m-2)}{3}\left(a(\tau)+\sqrt{\varepsilon}\|\tilde{z}\|_{\infty}\right)^{m-3} \varepsilon \int_{0}^{1} \tilde{z}_{x x}^{2} d x, \\
&=C^{\prime \prime}(m, \tau, \varepsilon) \int_{0}^{1} \tilde{z}_{x x}^{2} d x,
\end{aligned}
$$

where

$$
C^{\prime \prime}(m, \tau, \varepsilon):=\left(C^{\prime} \frac{(m-1)(m-2)}{3}\left(a(\tau)+\sqrt{\varepsilon}\|\tilde{z}\|_{\infty}\right)^{m-3} \varepsilon-(a(\tau))^{m-1}\right) .
$$

For $\tau>0$, we have

$$
C^{\prime \prime}(m, \tau, \varepsilon)<0
$$

if $\varepsilon$ is small enough.

From these estimates, we deduce that the $H^{1}$-norm is non increasing in the interval $\left(\tau_{1}, \tau_{2}\right)$. Hence, for all $\rho \geq 0$, we can choose $\varepsilon$ small enough to get $\left\|\tilde{z}\left(\tau_{2}\right)\right\|_{H^{1}(0,1)} \leq \varepsilon\left\|y_{1}\right\|_{H^{1}(0,1)} \leq \rho$.

\section{End of the proof of the main theorem}

We now go back to problem (3.4) but with Dirichlet boundary conditions and initial data $\tilde{z}\left(\tau_{2}\right)$ and apply an extension method that can be found, for instance, in [19, Chapter 2]. This method consists in extending the space domain from $(0,1)$ to $E:=(-d, 1+d)$ and inserting a sparse control in $\omega$, an nonempty open interval whose closure in $\mathbb{R}$ is included in $(-d, 0)$. We look at the following system

$$
\begin{cases}w_{t}-\left(m(1+\sqrt{\varepsilon} w)^{m-1} w_{x}\right)_{x}=\chi_{\omega} \tilde{u}, & (x, \tau) \in Q^{\prime}, \\ w(-d, \tau)=0, \quad w(1+d, \tau)=0, & \tau \in\left(\tau_{2}, \tau_{3}\right), \\ w\left(x, \tau_{2}\right)=w_{2}(x), & x \in E,\end{cases}
$$

where $Q^{\prime}=E \times\left(\tau_{2}, \tau_{3}\right)$ and $\tau_{3}=t_{3} / \varepsilon^{m-1}$. The function $w_{2} \in H_{0}^{1}(E) \cap$ $H^{2}(E)$ is an extension of $\tilde{z}\left(\tau_{2}\right)$ to $E$ which does not increase the $H^{1}$-norm, i.e. $\left\|w_{2}\right\|_{H^{1}(E)} \leq k\left\|\tilde{z}\left(\tau_{2}\right)\right\|_{H^{1}(0,1)} \leq \sqrt{\varepsilon} k\left\|y_{1}\right\|_{H^{1}(0,1)}$, for some $k>0$ independent of $\tilde{z}\left(\tau_{2}\right)$. 
Proposition 4.1. There exists $\rho>0$ such that, for any initial data $w_{2}$ with $\left\|w_{2}\right\|_{H^{1}} \leq \rho$ and for any $\varepsilon$ sufficiently small, system 4.1] is null controllable, i.e. there exists $\tilde{u} \in L^{2}\left(Q^{\prime}\right)$ such that $w\left(\tau_{3}\right)=0$.

Sketch of proof of Proposition 4.1. The proof is substantially the same as in 4. We just have to choose $\rho$ sufficiently small such that the solution of the control problem satisfies, for suitable value of $\varepsilon,\|w\|_{L^{\infty}}<\frac{1}{\sqrt{\varepsilon}}$.

Remark 4.2. Note that, combining the results in [4] and [16, pp. 360-364], the solution of (4.1) satisfies $w(0, \cdot), w(1, \cdot) \in H^{1}\left(\tau_{2}, \tau_{3}\right)$.

Proof of Theorem 1.1. We consider the function

$$
y(., t)= \begin{cases}y^{0}(., t), & t \in\left(0, t_{1}\right), \\ \frac{a(t)}{\varepsilon}+z(., t)=\frac{a(t)}{\varepsilon}+\frac{\tilde{z}(., t)}{\sqrt{\varepsilon}}, & t \in\left(t_{1}, t_{2}\right), \\ \frac{a(t)}{\varepsilon}+\frac{w(., t)}{\sqrt{\varepsilon}}, & t \in\left(t_{2}, t_{3}\right), \\ \frac{a(t)}{\varepsilon}, & t \in\left(t_{3}, T\right),\end{cases}
$$

which is a solution of system (1.4) with controls given by

$$
\begin{gathered}
u(t):=\frac{a^{\prime}(t)}{\varepsilon}, \quad t \in(0, T), \\
v_{0}(t):= \begin{cases}0, & t \in\left(0, t_{1}\right), \\
\frac{a(t)}{\varepsilon}+\frac{\tilde{z}(0, t)}{\sqrt{\varepsilon}}, & t \in\left(t_{1}, t_{2}\right), \\
\frac{a(t)}{\varepsilon}+\frac{w(0, t)}{\sqrt{\varepsilon}}, & t \in\left(t_{2}, t_{3}\right), \\
\frac{a(t)}{\varepsilon}, & t \in\left(t_{3}, T\right),\end{cases}
\end{gathered}
$$

and

$$
v_{1}(t):= \begin{cases}0, & t \in\left(0, t_{1}\right), \\ \frac{a(t)}{\varepsilon}+\frac{\tilde{z}(1, t)}{\sqrt{\varepsilon}}, & t \in\left(t_{1}, t_{2}\right), \\ \frac{a(t)}{\varepsilon}+\frac{w(1, t)}{\sqrt{\varepsilon}}, & t \in\left(t_{2}, t_{3}\right), \\ \frac{a(t)}{\varepsilon}, & t \in\left(t_{3}, T\right) .\end{cases}
$$

The function $y \in C\left([0, T] ; H^{-1}(0,1)\right)$ and as one can check using the improved regularity of the solution when it is strictly positive, $\left(v_{1}, v_{2}\right) \in H^{1}(0, T)^{2}$. So combining Proposition 2.4 Proposition 2.5 and Lemma 2.6. it is easy to see that the function given by $(4.2)$ is the solution on the interval $(0, T)$ of problem (1.4) with boundary conditions given by (4.4)- 4.5 and nonhomogeneous term 4.3$)$.

To conclude, we have for construction that $y(\cdot, T)=0$. 
The proof of part ii) follows the common argument of extension by symmetry. One first notice that using the smoothing property of 1.5 when $u=0$ and $v_{1}=0$, we may assume that $y_{0}$ is in $L^{2}(0,1)$. Then, we consider the auxiliary problem

$$
P_{D D}^{s} \begin{cases}y_{t}-\left(y^{m}\right)_{x x}=\widetilde{u}(t) \chi_{I}(t) & \text { in }(-1,1) \times(0, T), \\ y(-1, t)=v_{0}(t) \chi_{I}(t) \text { and } y(1, t)=v_{1}(t) \chi_{I}(t) & t \in(0, T), \\ y(x, 0)=\widetilde{y}_{0}(x) & x \in(-1,1),\end{cases}
$$

with $\widetilde{y}_{0} \in L^{2}(-1,1)$ defined by

$$
\widetilde{y}_{0}(x)=y_{0}(x) \text { and } \widetilde{y}_{0}(-x)=y_{0}(x), \forall x \in(0,1) .
$$

and with $v_{0}(t)=v_{1}(t)$. We apply the arguments of part i) to $P_{D D}^{s}$ with $(0,1)$ replaced by $(-1,1)$ and adjusting the formulation of $(4.1)$ in such a way that the control region $\omega$ is now symmetric with respect to $x=0$. Then, as we show later, the restriction of the solution of $P_{D D}^{s}$ to the space interval $(0,1)$ is the sought trajectory for system $P_{D N}$.

Lemma 4.3. Let $\omega$ be a nonempty open subset of $[-1-d, 1+d] \backslash[-1,1]$ which is symmetric with respect to $x=0$. Then, if $w_{2}$ is symmetric with respect to $x=0$, we can find a control $u_{s}$, symmetric w.r.t. $x=0$, such that the solution $w$ of system 4.1 satisfies

1. $w$ is symmetric w.r.t. $x=0$,

2. $w\left(\cdot, \tau_{3}\right)=0$.

Proof. The proof follows almost straightforward from [4, Theorems 4.1 and 4.2]. We just have to minimize the functional which appear in [4, Theorems 4.1] in the space of $L^{2}$ functions which are symmetric w.r.t. $x=0$.

The symmetry of the initial value implies as a consequence the symmetry of the solution $w$.

To conclude the proof of part ii) of Theorem 1.1 we note that as the solution $y(\cdot, t)$ of 4.6$)$ belongs to $H^{2}(-1,1)$ for all $t \in(0, T)$, we see that $y_{x}(0, t)=0$ for all $t \in(0, T)$ and so the conclusion is a direct consequence of part $\mathrm{i})$.

\section{Acknowledgments}

The research of Tommaso Mingazzini and Jesús Ildefonso Díaz has received funding from the ITN FIRST of the Seventh Framework Programme of the European Community (grant agreement number 238702). Jean-Michel Coron and Abdelmalek Drici were partially supported by the ERC advanced grant 266907 (CPDENL) of the 7th Research Framework Programme (FP7). Jesús Ildefonso Díaz has been partially supported also by DGISPI of Spain (ProjectMTM201126119) and the Research Group MOMAT (Ref. 910480) supported by UCM.

\section{References}

[1] Hans Wilhelm Alt and Stephan Luckhaus. Quasilinear elliptic-parabolic differential equations. Math. Z., 183(3):311-341, 1983. 
[2] Stanislav Antontsev, Jesús Ildefonso Díaz, and Sergey Shmarev. Energy methods for free boundary problems. Progress in Nonlinear Differential Equations and their Applications, 48. Birkhäuser Boston Inc., Boston, MA, 2002. Applications to nonlinear PDEs and fluid mechanics.

[3] Grigory Isaakovich Barenblatt. On some unsteady motions of a liquid and gas in a porous medium. Akad. Nauk SSSR. Prikl. Mat. Meh., 16:67-78, 1952.

[4] Marius Beceanu. Local exact controllability of the diffusion equation in one dimension. Abstr. Appl. Anal., (14):793-811, 2003.

[5] H. Brezis. Propriétés régularisantes de certains semi-groupes non linéaires. Israel J. Math., 9:513-534, 1971.

[6] Haïm Brézis. Monotonicity methods in Hilbert spaces and some applications to nonlinear partial differential equations. In Contributions to nonlinear functional analysis (Proc. Sympos., Math. Res. Center, Univ. Wisconsin, Madison, Wis., 1971), pages 101-156. Academic Press, New York, 1971.

[7] Haïm Brézis. Opérateurs maximaux monotones et semi-groupes de contractions dans les espaces de Hilbert. North-Holland, 1973.

[8] Marianne Chapouly. Global controllability of nonviscous and viscous Burgers-type equations. SIAM J. Control Optim., 48(3):1567-1599, 2009.

[9] Jean-Michel Coron. Global asymptotic stabilization for controllable systems without drift. Math. Control Signals Systems, 5(3):295-312, 1992.

[10] Jean-Michel Coron. On the controllability of 2-D incompressible perfect fluids. J. Math. Pures Appl. (9), 75(2):155-188, 1996.

[11] Jean-Michel Coron. Control and Nonlinearity, volume 136 of Mathematical Surveys and Monographs. American Mathematical Society, Providence, RI, 2007.

[12] Gregorio Díaz and Jesús Ildefonso Díaz. Finite extinction time for a class of nonlinear parabolic equations. Comm. Partial Differential Equations, 4(11):1213-1231, 1979.

[13] Jesús Ildefonso Díaz and Ángel Manuel Ramos. Positive and negative approximate controllability results for semilinear parabolic equations. Rev. Real Acad. Cienc. Exact. Fís. Natur. Madrid, 89(1-2):11-30, 1995.

[14] Jesús Ildefonso Díaz and Ángel Manuel Ramos. Approximate controllability and obstruction phenomena for quasilinear diffusion equations. Computational Science for the 21st Century (M.-O. Bristeau, G. Etgen, W. Fitzgibbon, J.-L. Lions, J. Periaux y M. F. Wheeler, eds.), John Wiley and Sons, Chichester, pages 698-707, 1997.

[15] Jesús Ildefonso Díaz and Ángel Manuel Ramos. Un método de viscosidad para la controlabilidad aproximada de ciertas ecuaciones parabólicas cuasilineales. Actas de Jornada Científica en homenaje al Prof. A. Valle Sánchez, (T. Caraballo et al. eds.), Universidad de Sevilla, pages 133-151, 1997. 
[16] Lawrence Craig Evans. Partial differential equations, volume 19 of Graduate Studies in Mathematics. American Mathematical Society, Providence, RI, second edition, 2010.

[17] Caroline Fabre, Jean-Pierre Puel, and Enrike Zuazua. Approximate controllability of the semilinear heat equation. Proc. Roy. Soc. Edinburgh Sect. A, 125(1):31-61, 1995 .

[18] Ján Filo. A nonlinear diffusion equation with nonlinear boundary conditions: method of lines. Math. Slovaca, 38(3):273-296, 1988.

[19] Andrei V. Fursikov and Oleg Yu Imanuvilov. Controllability of evolution equations, volume 34 of Lecture Notes Series. Seoul National University Research Institute of Mathematics Global Analysis Research Center, Seoul, 1996.

[20] Jacques Henry. Etude de la contrôlabilité de certains équations paraboliques. Thèse d'État, Université de Paris VI, 1978.

[21] Jacques-Louis Lions. Quelques méthodes de résolution des problèmes aux limites non linéaires. Dunod, 1969.

[22] Juan Luis Vázquez. The porous medium equation. Oxford Mathematical Monographs. The Clarendon Press Oxford University Press, Oxford, 2007. Mathematical theory. 